\title{
Der Vorrang der Selbstbestimmung
}

\section{Zur fundamentaltheologischen Kritik am Urteil des Bundesverfassungsgerichts zum assistierten Suizid}

\section{The primacy of self-determination \\ On the fundamental theological criticism of a judgment of the Federal Constitutional Court on assisted suicide}

\section{Matthias Tichy, Hamburg}

Zusammenfassung: Das jüngste Urteil des Bundesverfassungsgerichts zur Beihilfe zur Selbsttötung ist bei Kirchen und Ärztevertretern auf deutliche Ablehnung gestoBen. Auch der ehemalige Vorsitzende des Deutschen Ethikrats hat das Urteil scharf kritisiert. Die Vehemenz dieser Kritik deutet darauf hin, dass sich der Gegensatz zwischen Verteidigern und Gegnern des Vorrangs der Selbstbestimmung einer Person im Hinblick auf ihr eigenes Lebensende verhärtet hat. Um einen Ausweg aus diesem Patt zu finden, muss die Streitfrage in anderen Begriffen formuliert werden als in denen des Rechts und der kategorischen Gebote. Wichtige Hinweise dazu liefern Ronald Dworkins Überlegungen zur Ursache moralischer Konflikte.

Schlagwörter: Selbstbestimmung, Verfassungsgericht, öffentlicher Vernunftgebrauch, assistierter Suizid, Ronald Dworkin

Abstract: The recent judgement of the Federal Constitutional Court on assisted suicide has met with clear rejection by churches and medical representatives. The former chairman of the German Ethics Council has also sharply criticized the judgement. The vehemence of this criticism indicates that the contrast between defenders and opponents of the primacy of a person's self-determination with regard to the end of his or her own life has hardened. In order to find a way out of this stalemate, the issue must be formulated in terms other than those of rights and categorical requirements. Ronald Dworkin's reflections on the causes of moral conflict provide important pointers in this regard. 
Keywords: self-determination, constitutional court, public use of reason, assisted suicide, Ronald Dworkin

In einer ersten Reaktion auf die Aufhebung des Verbots der „geschäftsmäBigen Förderung der Selbsttötung“ durch das Bundesverfassungsgericht im Februar 2020 (BVerfG 2020) hat der damalige Vorsitzende des Deutschen Ethikrats, Peter Dabrock, kritisiert, dieses Urteil verkehre alles, „was das Gericht bislang über Menschenwürde gesagt hat“. Es gehe von einem „völlig überhöhten Autonomiebegriff“ aus, gegenüber dem der Schutz des Lebens eines Menschen „nichts“ wiege. Die Waage neige sich „bis zum Anschlag in Richtung grenzenloser Autonomie“ (Dabrock 2020).

Durch die Berichterstattung in den Medien entstand tendenziell der Eindruck, als spreche Dabrock für den Ethikrat als ganzen. Aber seine Kritik ist zunächst einmal eine persönliche Stellungnahme, was sich schon daraus ergibt, dass sie bereits am Tag nach der Urteilsverkündung vorlag. Was die Frage der Selbstbestimmung am Lebensende betrifft, ist es außerdem gar nicht möglich, die Auffassung des Ethikrats insgesamt zu vertreten, weil sich seine Mitglieder in den bisherigen Stellungnahmen und Empfehlungen zu diesem Problemkreis nicht auf ein einheitliches Votum verständigen konnten. Im Kern blieb es bei der Feststellung der Unvereinbarkeit entgegengesetzter Positionen, nämlich einerseits, dass die Pflicht zur Bewahrung menschlichen Lebens im Konfliktfall Vorrang vor dem Selbstbestimmungsrecht haben soll, und andererseits, dass das Recht auf Selbstbestimmung überwiegt. ${ }^{1}$ Dementsprechend bleibt auch abzuwarten, ob der Ethikrat zu einer einheitlichen Stellungnahme gelangen wird, was die Kernsätze aus dem Urteil des Bundesverfassungsgerichts vom Februar 2020 betrifft. Es spricht einiges dafür, dass eine solche Erwartung verfehlt wäre. Deshalb gehe ich im ersten Teil kurz auf die Frage ein, worin die Aufgabe des Ethikrats in einer pluralistischen Gesellschaft bestehen kann und welche Ergebnisse von ihm zu erwarten sind.

Unter der Vielzahl von Reaktionen auf das Verfassungsgerichtsurteil hebt sich Dabrocks Kritik dadurch hervor, dass sie aus dem Kontext meh-

1 Vgl. Ethikrat 2006, 81-82 und 96-105, sowie Ethikrat 2014, 3. Dabrock wurde erst 2012 in den Ethikrat berufen, vertritt jedoch Positionen, die denjenigen der Mehrheit der Mitglieder des Ethikrats im Jahr 2006 vergleichbar sind. 
rerer Beiträge zu verstehen ist, in denen Dabrock seine fundamentaltheologische Position ausgearbeitet hat. Sie bildet einen paradigmatischen Fall für Auffassungen, die in medizin- oder bioethischen Debatten von Seiten derer vertreten werden, für die das Recht auf individuelle Selbstbestimmung durch Pflichten „gegen sich selbst“ begrenzt wird. Eine Auseinandersetzung mit Dabrocks Position ist daher von generellem Interesse, und deshalb untersuche ich im zweiten Teil die Argumentation, auf die Dabrock seine Ablehnung des Verfassungsgerichtsurteils stützt. Ein Grund für den Dissens liegt darin, dass unterschiedliche Bedeutungen des Begriffs Selbstbestimmung vorausgesetzt werden. So ergibt sich ein argumentatives Patt zwischen den Vertretern der „konservativen“ Position, die das kategorische Gebot des Lebensschutzes auch gegen den Willen des Betroffenen verteidigen, und den Vertretern einer „liberalen“ Auffassung, die den Vorrang personaler Autonomie behaupten. Im dritten Teil schlage ich im Anschluss an Ronald Dworkin eine Strategie vor, die einen Ausweg aus dieser Pattsituation eröffnen könnte. Zwar sind die Arbeiten Dworkins zu medizinethischen Problemen bereits vor einigen Jahren erschienen (vgl. Dworkin 1994 und 2014). Seine Überlegungen zum Umgang mit festgefahrenen argumentativen Konflikten sind aber weiterhin aktuell und bieten sich als Grundlage für die Ausarbeitung entsprechender methodischer Regeln an.

Laut Gesetz soll der Ethikrat „Empfehlungen für politisches und gesetzgeberisches Handeln“ erarbeiten (§2, 1 EthRG) und dabei „unterschiedliche ethische Ansätze und ein plurales Meinungsspektrum“ berücksichtigen (§4, 2 EthRG). Einerseits soll der Realität einer pluralistischen Gesellschaft Rechnung getragen, andererseits sollen Empfehlungen gegeben werden, die einen gewissen Grad von Eindeutigkeit aufweisen müssen, um brauchbar zu sein. In ihren bisherigen Stellungnahmen und Empfehlungen konnten die Mitglieder des Ethikrats allerdings keinen Konsens in der Frage erzielen, über die das Verfassungsgericht zu entscheiden hatte, nämlich in der Frage der Zulässigkeit der Beihilfe zur Selbsttötung. Was als „Stellungnahme“ zum Thema „Selbstbestimmung und Fürsorge am Lebensende“ veröffentlicht wurde, ist in Wirklichkeit eine Dokumentation der offenen Auseinandersetzung über die Frage, ob im Konfliktfall dem Selbstbestimmungsrecht oder dem Lebensschutz der Vorrang einzuräumen ist (Ethikrat 2006, 81-82). Die Vertreter beider Auffassungen kommen abschließend zwar zu einigen ge- 
meinsamen Empfehlungen für rechtliche Regelungen, aber daran ist wiederum ein „ergänzendes Votum“ einer Minderheit angefügt, das diesen Empfehlungen in wichtigen Punkten widerspricht (Ethikrat 2006, 96-101 bzw. 103-105). Auch über die „Ad-hoc-Empfehlung“ anlässlich der Entscheidung des Bundesverwaltungsgerichts vom März 2017 über die Zulässigkeit der Abgabe eines Betäubungsmittels an Sterbewillige durch staatliche Stellen gab es keinen Konsens (BVerwGE 2017). Eine Mehrheit von 16 Mitgliedern lehnte das Urteil ab, eine Minderheit von neun Mitgliedern hielt es für „ethisch wohl erwogen und begrüßenswert“ (Ethikrat 2017, 3). ${ }^{3}$

Aus der Tatsache dieser Dissense allein ergibt sich noch kein Grund zur Kritik. Aber ein Dissens ist auch keine unausweichliche Konsequenz des Faktums des Pluralismus, soweit es sich in der Zusammensetzung der Mitglieder des Ethikrats widerspiegelt. Dem Ethikrat sollen außer Wissenschaftlerinnen und Wissenschaftlern auch „anerkannte Personen an[gehören], die in besonderer Weise mit ethischen Fragen der Lebenswissenschaften vertraut sind“ ( $\$ 4,1$ EthRG). In der Realität bestimmen Bundesregierung und Bundestag unter anderen zwei Repräsentanten der Kirchen sowie je einen Vertreter der muslimischen und der jüdischen Glaubensgemeinschaft (Gethmann 2020, 32). Von ihnen wird aber ebenso wie von den berufenen Wissenschaftlern erwartet, dass sie konkurrierende Auffassungen aus ihrem Fachgebiet zur Sprache bringen und die jeweils eigene Auffassung als eine unter anderen darzustellen bereit sind. Anders als ein Gericht oder ein Parlament muss der Ethikrat zwar nicht in jedem Fall zu einer Entscheidung gelangen. Jedoch hat ein Dissens unterschiedliche Gründe und eine unterschiedliche Bedeutung, je nachdem, ob der Ethikrat überwiegend als „Rat der Stellvertreter“ oder als „Rat der Sachverständigen“ (Gethmann 2020, 32f.) agiert. Als Vertreter unterschiedlicher Weltanschauungen und Interessen werden die Mitglieder des Rats in einem pragmatisch-politischen Diskurs versuchen, eine mehrheitsfähige Auffassung zu entwickeln, und ihre Position dementsprechend anpassen. Hingegen sollen Experten in einem „Rat der Sachverständigen“ Prämissen und Konsequenzen konfligierender

2 Dieses Urteil nimmt in wesentlichen Teilen das Urteil des Verfassungsgerichts vorweg. In der Begründung wird aus dem allgemeinen Persönlichkeitsrecht (Art. 2, 1 GG in Verbindung mit Art. 1, 1 GG) das Recht zur Entscheidung über das Ende des eigenen Lebens abgeleitet (BVerwGE 2017).

3 In der Pressemitteilung 4/2017 des Ethikrats (1.6.2017) heißt es, dass der Deutsche Ethikrat dem Urteil des Bundesverwaltungsgerichts „widerspricht“. 
Auffassungen so weit transparent machen, dass erkennbar wird, in welchen Punkten ein begründeter Konsens besteht oder auf welchem Weg er erreicht werden könnte (vgl. van den Daele 2008, 371ff.; Quante 2014, 85ff.). Im Hinblick auf das Ziel der Erarbeitung von Empfehlungen an die Politik sollte der Ethikrat also Kontroversen, die in öffentlichen Diskursen ausgetragen werden, nicht stellvertretend wiederholen, sondern deren Voraussetzungen analysieren und neue Gesichtspunkte entwickeln, um verhärtete Gegensätze zu überwinden.

Aber darum geht es Dabrock, wie seine Urteilsschelte zeigt, in diesem Fall offenbar nicht. Er zieht nicht einmal in Erwägung, dass das Bundesverfassungsgericht mit der Betonung der „persönlichen Autonomie“ als obersten Bezugspunktes das Ziel verfolgt haben könnte, eine Interpretation der Verfassung zu liefern, die die Kohärenz der Rechtsordnung bestmöglich wahrt. Stattdessen wirft Dabrock dem Gericht einen „radikale[n] Bruch mit der bewährten Rechtskultur“ vor und fordert eine „politische und gesellschaftliche Antwort“von Seiten derer, die nicht wollen, dass „unsere Rechtsordnung so schrankenlos ist“ (Dabrock 2020), wie das Verfassungsgericht es Dabrock zufolge zulässt.

Wenn das Urteil einem „radikalen Bruch“ mit der bisherigen Rechtsprechung gleichkäme, wären dem Verfassungsgericht allerdings elementare handwerkliche Mängel vorzuwerfen. Zu begründen wäre ein solcher Vorwurf aber nur durch den Nachweis, dass das Gericht seine Aufgabe nicht erfüllt hat, geltendes Recht auszulegen, in diesem Fall ein Gesetz auf Verfassungsgemäßheit zu prüfen. Anhaltspunkte dafür liefert Dabrock in seiner Stellungnahme und auch an anderer Stelle aber nicht. Seine Kritik erfolgt aus der Perspektive der (evangelisch-theologischen) Ethik und bleibt außerhalb des Bereichs juridischer Erwägungen. Aber auch unter ethischen Aspekten ist sie auf ihre Stimmigkeit zu prüfen.

Dabrock versteht seinen „fundamentaltheologischen“ Ansatz als „Beitrag zur Gestaltung des öffentlichen Vernunftgebrauchs“ (Dabrock 2010, 228) und nimmt damit einen Kerngedanken von John Rawls für seine Position in Anspruch. Um eine religiös-theologische Ethik mit dem Entwurf des politischen Liberalismus verbinden zu können, muss Dabrock aber erhebliche Veränderungen und Ergänzungen der Theorie von Rawls vornehmen. Bekanntlich zeichnet sich nach Rawls der öffentliche Vernunftgebrauch da- 
durch aus, dass grundlegende Fragen der politischen Verfassung verhandelt werden, und zwar unter „vernünftiger Abwägung öffentlicher politischer Werte“ (Rawls 1998, 348). Sowohl für den Gegenstand der Verhandlung als auch für die Art der Gründe, die angeführt werden dürfen, gibt Rawls präzise Bedingungen an. ${ }^{4}$ Diese Voraussetzungen übernimmt Dabrock nicht, so dass sich die Bedeutung des Begriffs verschiebt. Dies gilt auch für seinen Gebrauch des Begriffs des „übergreifenden Konsenses“. Rawls führt ihn ein, um die Möglichkeit der „Versöhnung durch öffentlichen Vernunftgebrauch“ zu erklären. Ein Konsens über Gerechtigkeitsgrundsätze kann nur gelingen, wenn die „öffentliche Gerechtigkeitskonzeption [...] soweit wie möglich losgelöst von umfassenden religiösen, philosophischen und moralischen Lehren vorgestellt" (Rawls 1998, 232) wird. Weil diese Lehren unvereinbare Vorstellungen vom „guten Leben“ enthalten, darf sich die Begründung einer Gerechtigkeitskonzeption, wenn sie allgemein akzeptierbar sein soll, nicht auf spezifische Elemente aus diesen Lehren stützen. Über deren Differenzen hinweg kann aber ein Konsens über die Grundstruktur einer gerechten Gesellschaft erreicht werden, eben ein „übergreifender Konsens“ („overlapping consensus").

Aber Dabrock geht es nicht um die Bestimmung von Verfassungsgrundsätzen oder Grundrechten, sondern um die Verteidigung der These, dass „Ethikansätze, die ausdrücklich kulturell geprägt sind und bleiben sollen, in der Begegnung mit Fremdem über sich hinausgeführt werden und sich so [...] auf die Suche nach einem overlapping consensus begeben“, wobei sie aber „ihre eigene Deutungsperspektive“ (Dabrock 2004a, 22f.) bewahren. Während eine Universalisierung durch Abstraktion lediglich zu einer Minimalmoral oder zu einem formalen System gleicher Rechte führt, sollen in diesem „laterale[n] Universalisierungsverfahren“ materiale Traditionen daraufhin befragt werden, ob sich „Begründungscluster aus diesen verschiedenen Perspektiven entdecken lassen“" (Dabrock 2004a, 42f.). Diese Begründungen betreffen sowohl Gerechtigkeitsfragen als auch Fragen des guten Lebens, denn „Gerechtes und Gutes lassen sich nicht messerscharf trennen“ (Dabrock 2004a, 34).

4 Rawls hat später eine „weite“ Sicht des öffentlichen Vernunftgebrauchs eingeführt, der zufolge durchaus „vernünftige Globaltheorien“ in den Diskurs eingebracht und erörtert werden können, jedoch müssen sich Vorschläge zur Gesetzgebung auch durch öffentliche Gründe oder durch Hinweis auf anerkannte politische Werte begründen lassen (vgl. Rawls 2003, 146). Auch mit dieser „weiten“ Sicht ist Dabrocks Konzeption nicht vereinbar. 
Nur aufgrund dieser erheblichen Bedeutungsverschiebungen kann Dabrock die Begriffe des öffentlichen Vernunftgebrauchs und des übergreifenden Konsenses mit seinem fundamentaltheologischen Ansatz verbinden und (gegen Rawls) daran festhalten, dass aus religiös-theologischer Perspektive trotz des partikularen Ausgangspunkts ein essentieller Beitrag gemäß den Bedingungen des öffentlichen Vernunftgebrauchs geleistet werden kann. Die Einbeziehung religiöser Beiträge soll dadurch möglich werden, dass die fundamentaltheologische Ethik „Differenzsensibilität“ entwickelt und sich aufgrund der „Anerkennung der Begrenztheit des Eigenen“ (Dabrock 2004a, 41) gegenüber anderen Ethikentwürfen öffnet. Es muss hier offen bleiben, wie eine solche „Transpartikularisierung“ (Dabrock 2004a, 42ff.) im Einzelnen verlaufen soll und ob sie überhaupt möglich ist. Jedenfalls entschärft Dabrock vorsorglich das Problem unvereinbarer Gegensätze durch den Hinweis, dass Anhänger anderer Lehren „aus guten Gründen [...] zu anderen Urteilen kommen können“ als den jeweils eigenen. In diesem Fall sei „auf unausweisbare moralische Eindeutigkeitsprätentionen“ zu verzichten, damit „nicht die moralische Kommunikation als solche abbricht“. So ergebe sich ein „realistischer Pluralismus im Anwendungsbezug von Ethik“ (Dabrock 2004a, 54).

Was das Verhältnis von Selbstbestimmung und Lebensschutz angeht, zieht Dabrock jedoch eine klare Grenze: „Selbstverständlich herrscht [...] in der evangelischen Ethik kein Pluralismus hinsichtlich der Anerkennung [...] der Würde des Anderen und der grundsätzlichen Schutzwürdigkeit menschlichen Lebens“ (Dabrock 2004a, 53). Als Bedingung der Würde sei das Leben ebenso zu schützen wie diese selbst (Dabrock 2004b, 118). Weil die Verpflichtung zum Lebensschutz grundsätzlich auch für den Sterbenswilligen gilt, ist seiner Selbstbestimmung in diesem Punkt eine Grenze gesetzt. Dagegen räumt das Verfassungsgericht dem Selbstbestimmungsrecht den Vorrang ein, woraus sich der Grund für Dabrocks Kritik am Urteil des Gerichts ergibt.

Den ethischen Pluralismus, den Dabrock auf programmatischer Ebene befürwortet, setzt er auf der pragmatischen Ebene des Argumentierens nicht um. Er lässt keine Bereitschaft erkennen, die liberale Position vom Vorrang des Selbstbestimmungsrechts wenn nicht als richtigen, so doch als ernstzunehmenden moralischen Standpunkt zu akzeptieren. ${ }^{5}$ Vermutlich

5 Der „Herausforderung liberaler Ethiken“ (Dabrock 2004a, 23) begegnet Dabrock mit dem Hinweis auf die gesellschaftlich-geschichtlichen Hintergründe 
liegt der Grund für diese Diskursbeschränkung darin, dass die Annahme der kategorischen Geltung eines Gebots mit der Argumentationsregel verwechselt wird, über diese Voraussetzung sei eine Auseinandersetzung weder möglich noch erforderlich. In diesem Fall fungiert der Verweis auf die Menschenwürde nicht als Argument auf der Inhaltsebene, sondern markiert eine Grenze für die Auseinandersetzung; die Unantastbarkeit menschlicher Würde verwandelt sich unter der Hand in die Unangreifbarkeit der Position desjenigen, der sich auf sie beruft. Aber auch wenn ein Konsens über die kategorische Geltung eines Gebots besteht, kann es noch Kontroversen über seinen präzisen Gehalt oder seine Anwendbarkeit auf einen konkreten Fall geben. Die Angemessenheit der Berufung auf ein Gebot muss in einer diskursiven Auseinandersetzung erst noch begründet werden. Hinsichtlich seines präzisen Gehalts und seiner Anwendbarkeit ist also durchaus ein Pluralismus der Auffassungen über das Lebensschutzgebot gegeben, so dass der Gegensatz zwischen der konservativen und der liberalen Auffassung weniger scharf sein könnte, als er auf den ersten Blick erscheint.

Ein unverzichtbares Argument für Dabrocks Überlegungen ist die Annahme, dass sich aus dem Menschenwürdegrundsatz das Gebot des Lebensschutzes ergibt. Gegen diese „Kopplung“ von Menschenwürde und Lebensschutz wurden rechts- und moralphilosophische Einwände vorgebracht (z. B. Dreier 2007, 319; Quante 2014, 47f.). Die Debatte um das Verhältnis von Würde und Lebensschutz ist zu umfangreich, um sie weiter zu verfolgen. Außerdem besteht die Gefahr, mit einem weiteren Beitrag lediglich zur Verfestigung des Gegensatzes zwischen unvereinbaren Positionen beizutragen. Diese Gefahr lässt sich am Verlauf der Debatte um die Präimplantationsdiagnostik studieren. Damals habe es, so fasst Michael Quante zusammen, zunächst eine „Entideologisierung und Versachlichung“ des Diskurses gegeben, aber anschließend sei der „Rationalisierungsprozess unserer Diskussionskultur [...] rückläufig“ (Quante 2014, 203; vgl. 28) gewesen. Den Grund dafür sieht Quante in der Fokussierung der Auseinandersetzung auf die Frage der ,ethischen Zulässigkeit der Lebensqualitätsbewertung“ (Quante 2014, 205). Solange eine prinzipielle Unvereinbarkeit von Menschenwürde und Lebensqualitätsbewertung angenommen wird, lässt sich mit dem Verweis

des konkreten moralischen Urteils und dessen Bindung an das allgemeine Sittengesetz (Dabrock 2004a, 28-29). Aber dieser Blick von außen auf die Genese und den Gehalt einer Gewissensentscheidung ändert nichts daran, dass der Primat der Selbstbestimmung verlangt, die Entscheidung einer Person, die nur deren Belange betrifft, zu respektieren. 
auf die Menschenwürde jede Argumentation abblocken, die Aussagen über Lebensqualität enthält. Ebenso kann sich die Auseinandersetzung über die Zulässigkeit der Beihilfe zur vorzeitigen Beendigung des eigenen Lebens als unproduktiv erweisen, wenn sie im Streit über die Unvereinbarkeit mit dem Menschenwürdeprinzip endet, aus dem wiederum das Gebot des Lebensschutzes folgen soll. Um einen Ausweg aus dieser argumentativen Pattsituation zu finden, muss das Problem in einem anderen begrifflichen Rahmen neu gestellt werden.

Die Dringlichkeit dieser Reformulierung ergibt sich auch daraus, dass einige Verteidiger des kategorischen Lebensschutzgebots selbst die Möglichkeit von Konfliktsituationen einräumen, in denen es unangemessen wäre, die Beweggründe eines Sterbewilligen unter Verweis auf das Lebensschutzgebot schlicht zu übergehen (z. B. Dabrock 2015, 215). Dennoch wollen sie an der kategorischen Geltung des Lebensschutzgebots festhalten. Das lässt keine schlüssige Begründung ihrer Auffassung zu, wie die folgende Zusammenfassung der Argumentationsschritte zeigt: ${ }^{6}$

(1) Das Gebot des Schutzes der Menschenwürde gilt kategorisch.

(2) Aus dem Menschenwürdegrundsatz lässt sich das Gebot des Lebensschutzes ableiten, das daher ebenfalls kategorisch gilt. (Menschliches) Leben ist nicht gegen andere Werte abzuwägen.

(3) Die personale Autonomie im Sinn der Fähigkeit, das eigene Leben entsprechend einer eigenen Vorstellung vom guten Leben und Lebensende zu führen, ist unter gewöhnlichen Voraussetzungen zu respektieren.

(4) Aus der Kombination von (2) und (3) ergibt sich, dass der Anspruch auf Autonomie zurücktreten muss, wenn das Leben eines Menschen nur gegen seinen erklärten und wohlüberlegten Willen geschützt werden kann.

(5) Wenn der Wunsch eines Sterbewilligen nachvollziehbar ist und nicht unter Hinweis auf andere Handlungsoptionen abgewiesen werden kann, ergibt sich ein Dilemma. Einerseits soll die Pflicht zum Lebensschutz (2) kategorisch gelten, andererseits verbietet es der Respekt vor dem Sterbewilligen, ihm in einer offensichtlichen Notlage Hilfe zu verweigern. Obwohl es einem Verstoß gegen ein kategorisches Gebot

6 Der folgende Versuch einer Zusammenfassung und Rekonstruktion stützt sich auf verschiedene Beiträge, insbesondere auf Braun, Ried und Dabrock 2015; Dabrock 2004a, 2004b, 2015; Schardien 2004; Ethikrat 2006, 2014 und 2017. 
gleichkommt, muss daher akzeptiert werden, dass es Ausnahmen von (4) gibt.

(6) Dabei muss die strikte Asymmetrie zwischen Regeln (2-4) und Ausnahme (5) gewahrt bleiben, da der Wert menschlichen Lebens (2) weiterhin als unabwägbar angesehen werden soll. Deshalb können keine Kriterien angegeben werden, mit deren Hilfe sich entscheiden ließe, ob ein Ausnahmefall vorliegt. So wird verhindert, dass es durch Berufung auf Präzedenzfälle zu einer „Normalisierung“ der Beihilfe zur Selbsttötung kommt.

Die Zusammenfassung der Argumentationsschritte verdeutlicht den Versuch, um den Preis einer kaum zu verbergenden Inkohärenz das kategorische Gebot des Lebensschutzes mit der intuitiv plausiblen und durch Erfahrungen gestützten Annahme zu vereinbaren, eine Beihilfe zur Selbsttötung könne unter spezifischen Voraussetzungen erlaubt oder sogar geboten sein. Einerseits wird die kategorische Geltung des Lebensschutzgebots behauptet (2), andererseits wird auf die Möglichkeit von Ausnahmen hingewiesen (5). Um einen offenen Widerspruch und damit das Scheitern der Argumentation zu verhindern, wird hervorgehoben, dass eine Ausnahme allenfalls in einer Grenzsituation, in einem „existentiellen und exzeptionellen ,Notstand““ (Schardien 2004, 307) in Betracht kommt. So bleibt das Gebot des Lebensschutzes, sozusagen ex negativo, das einzige Gebot, das für eine Entscheidung über Suizidbeihilfe relevant ist, so dass im Ausnahmefall eine „Schuldübernahme unausweichlich“ (Dabrock 2004b, 144) ist. Eine Gewissensentscheidung, gegen die Norm zu handeln, lasse sich höchstens respektieren, niemals aber rechtfertigen, und so werden auch die „üblichen Normkonsequenzen nicht [...] sistiert“ (Dabrock 2015, 130). ${ }^{7}$ Die Ausnahme bildet also keinen Sonderfall, für den abweichende Regeln gelten, sondern eine Anomalie, ein singuläres Ereignis, für dessen Beschreibung und Bewertung es keine angemessenen Kategorien gibt.

Damit die Ausnahme weiterhin als Anomalie isoliert bleibt und im öffentlichen Diskurs, wenn überhaupt, nur als marginaler Fall thematisiert wird, muss ihre „Normalisierung“ verhindert werden, und so ist die Warnung vor einer „Normalisierung der Suizidassistenz“ (Dabrock 2015, 218)

7 Dabrock kritisiert daher eine Mehrheitsentscheidung des Ethikrats, der eine „liberalisierende Änderung des Betäubungsmittelgesetzes“, nämlich eine Straffreiheit im Zusammenhang mit einer Suizidassistenz, vorgeschlagen hatte (Dabrock 2015, 132, Anm. 24). 
ein Leitmotiv der konservativen Auffassung. Unter „Normalisierung“ ließe sich zunächst eine rechtliche Regelung verstehen, mit der die Bedingungen spezifiziert würden, unter denen Suizidbeihilfe im Ausnahmefall zulässig ist. Die Bedenken richten sich aber nicht auf die rechtlichen Regelungen als solche, sondern auf die vermuteten Konsequenzen. Um die Warnung vor einer Normalisierung nachvollziehen zu können, sind daher verschiedene, wenn auch zusammenhängende Bedeutungen des Ausdrucks Normalisierung zu unterscheiden:

(a) Eine Normalisierung kann mit der Aufhebung eines Tabus zusammenfallen. Was zuvor nicht Gegenstand des öffentlichen Diskurses war, wird zum Thema.

(b) Eine Normalisierung kann darin bestehen, dass eine bisher ungeregelte Praxis ausdrücklich einer Regel, einer Norm unterworfen wird.

(c) Normalisierung kann bedeuten, dass eine Handlungsweise als übliche, gewohnheitsmäßige Praxis akzeptiert wird.

(d) Normalisierung kann heißen, dass eine zuvor seltene Handlungsweise häufiger zu beobachten ist.

In keiner der vier Bedeutungsvarianten ist eine Normalisierung als solche aus ethischen oder moralischen Gründen abzulehnen. Was (a) die Aufhebung eines Tabus angeht, so mag sie von einigen Personen bedauert werden, erweist sich aber oft als unaufhaltsam und ist im Hinblick auf die Frage des Rechts zur Selbsttötung und auch der Beihilfe dazu bereits weitgehend erfolgt. Ob die Aufhebung eines Tabus zu befürworten oder abzulehnen ist, hängt von seinem Gegenstand ab, nicht von der Enttabuisierung als solcher. Für diejenigen, die daran denken, gegen ein Tabu zu verstoßen, kann die Enttabuisierung eine Chance sein, über die eigene Konfliktsituation sprechen zu können. Ähnliches gilt für eine philosophische Auseinandersetzung: Sie verträgt sich in keinem Fall mit der Aufrechterhaltung eines Tabus.

Eine Normalisierung im Sinn einer rechtlichen Regelung (b) schafft Rechtssicherheit, wogegen zunächst einmal keine Bedenken geltend zu machen sind. Allenfalls im Hinblick auf die vermuteten weiteren Folgen (c) und (d) können sich Bedenken ergeben. Beispielsweise lehnte eine Mehrheit des Ethikrats eine rechtliche Regelung der (ärztlichen) Suizidassistenz ab mit der Begründung, hierdurch würden „gleichsam ,erlaubte Normalfälle“ [...] definiert" (Ethikrat 2014, 3). Anscheinend wurde angenommen, dass von dieser Option gewohnheitsmäßig und häufig Gebrauch gemacht würde. Aber selbst daraus folgt nicht, dass diese Praxis aus ethischen Gründen abzulehnen ist; diese Folgerung ergibt sich erst zusammen mit der zusätzlichen 
Voraussetzung, dass (i) diese Praxis bereits als solche und im Einzelfall moralisch falsch ist oder (ii) der (häufige) Vollzug bedenkenlos erfolgt oder (iii) die wahrscheinlichen Auswirkungen dieser Praxis negativ zu bewerten sind. Voraussetzung (i) ist trivial, da sie den beiden ersten Prämissen im oben angegebenen Argumentationsschema entspricht, aber dabei die Lücke wieder schließt, die der Hinweis auf die Möglichkeit einer existentiellen Ausnahmesituation geöffnet hat. Aus der Voraussetzung (ii) folgt, dass auch in dem Fall einer generell akzeptierten und verbreiteten Praxis die Handlungsverantwortung im Einzelfall bei denen liegt, die gemäß dieser Praxis handeln. Auch wenn die bedenkenlos Handelnden ihrer Verantwortung nicht gerecht werden, fällt nicht denjenigen die (volle) Verantwortung zu, die eine an sich legitime Praxis rechtlich regeln und unter bestimmten Bedingungen zulassen. Welche Auswirkungen eine Normalisierung in Form einer gesetzlichen Regelung auf das Verhalten der Menschen hat (iii), ist eine empirische Frage, die nicht vorgreifend entschieden werden kann. Dabrock hält es allerdings für eine „realistische Erwartung“, dass nach einer Liberalisierung der Regelung zur Suizidassistenz zunehmend Druck auf „vulnerable Gruppen“ ausgeübt würde, von der Option einer vorzeitigen Beendigung des eigenen Lebens Gebrauch zu machen (Dabrock 2015, 128). Angesichts dieser Gefahr müsse der Anspruch auf Suizidassistenz, auch wenn er sich aus dem Selbstbestimmungsrecht ergibt, zurücktreten (Dabrock 2015, 129).

Die Warnung vor einer Normalisierung hat für die Schlüssigkeit der Argumentation der Verteidiger des Vorrangs des Lebensschutzes eine zweifache Bedeutung: (1) Solange die Fälle, in denen eine Beihilfe zur Selbsttötung zulässig erscheint, als Ausnahme im Sinn einer Anomalie angesehen werden können, behält das Gebot des Lebensschutzes seinen (quasi) kategorischen Status. (2) Die Warnung vor einer Normalisierung setzt, sofern sie erfolgreich ist, dem öffentlichen Diskurs bestimmte Grenzen im Hinblick darauf, was thematisiert werden darf, und hat dadurch eventuell Einfluss auf politische Entscheidungen. Beide Konsequenzen sind unbefriedigend. Denn erstens (1) verdient die Frage, ob denn nun das Gebot des Schutzes menschlichen Lebens kategorisch - eventuell mit Ausnahmen für den Fall, dass Leben gegen Leben steht - gilt, eine klare Antwort. Zweitens (2) ist die Überzeugungskraft von Folgenargumenten generell begrenzt, weil Folgen nur mit einer gewissen Wahrscheinlichkeit eintreten. Außerdem hängen die befürchteten Auswirkungen einer Normalisierung mit von den Entscheidungen zahlreicher involvierter Personen und Institutionen ab. Schließlich sind die „gesellschaftlichen Kosten“ einer Quasi-Tabuisierung zu bedenken, auf 
die eine Begrenzung des Diskurses über die Zulässigkeit der Suizidbeihilfe hinausläuft. So spricht sich beispielsweise in Meinungsumfragen eine Mehrheit der Befragten unter Berufung auf das Selbstbestimmungsrecht offen für eine Liberalisierung der Gesetzgebung aus (Braun, Ried und Dabrock 2015, 219ff.). Daraus ziehen Braun, Ried und Dabrock keine direkten Konsequenzen, sondern relativieren stattdessen den Wert der Umfrage, indem sie dem Selbstbestimmungsrecht eine andere Deutung geben: Selbstbestimmung am Lebensende müsse von vornherein zusammen mit der Furcht vor dem Sterben und mit den Auswirkungen einer Liberalisierung der Suizidassistenz auf die „gesellschaftliche Kohäsion“ (Braun, Ried und Dabrock 2015, 221) gedacht werden.

Der Gegensatz zum Tenor des Verfassungsgerichtsurteils ist deutlich genug. In seiner Begründung des Urteils stützt sich das Gericht auf die Prämisse, dass ein „Akt autonomer Selbstbestimmung von Staat und Gesellschaft zu respektieren ist“ (BVerfG 2020, 1). Selbstbestimmung ist hier nicht als universell-vernünftige Selbstgesetzgebung im Sinn Kants zu verstehen, sondern im Sinn eines (normativen) Individualismus: Die „eigenverantwortliche Entscheidung über das eigene Lebensende [bedarf] keiner weiteren Begründung oder Rechtfertigung" (BVerfG 2020, 63 Rn. 210). Insbesondere ist niemand zur Rechtfertigung für Handlungen, die nur die eigene Person betreffen, im Hinblick auf gesellschaftliche Leitbilder oder religiöse Lehren verpflichtet. Dieses Verständnis von Selbstbestimmung als Befreiung von der Rechtfertigungspflicht für Entscheidungen über die eigenen Belange gilt im Hinblick auf die rechtlich-äußere Freiheit; im Kontext der ethischen Frage nach dem gelingenden Leben sind zusätzliche Gesichtspunkte zu berücksichtigen.

Sowohl unter rechtlichen wie unter ethischen Gesichtspunkten ist von Selbstbestimmung in normativer Hinsicht die Rede, das heißt, unabhängig von der Frage nach den Konstitutionsbedingungen oder der empirisch-realen Verfassung des „Selbst“. Vorauszusetzen ist lediglich, dass es empirisch identifizierbare Merkmale gibt, die einen guten Grund dafür abgeben, einen Menschen als Person anzusehen und ihm die Fähigkeit zur Selbstbestimmung zuzuschreiben. Zwischen dem normativen Begriff der (personalen) Autonomie als der „Fähigkeit zur rationalen Selbstbestimmung“ (Gutmann und Quante 2017, 331) und einem deskriptiv-phänomenologischen Begriff, der das Selbst in seiner Entwicklung und seinen sozialen Bezügen beschreibt, muss unterschieden werden.

Wenn Dabrock nun für ein „alternatives Selbstbestimmungsverständnis“ (Dabrock 2015, 125) plädiert, dem zufolge Selbstbestimmung aus der Be- 
ziehung des Selbst zu seinem Anderen zu verstehen ist, blendet er die normative Komponente aus. Er will Selbstbestimmung „beziehungsorientiert und inklusionssensibel ausdeuten“ und die Ergebnisse dann „in den Rahmenbedingungen des Staates umgesetzt sehen“ (Dabrock 2015, 126). Damit kommt zwar wieder ein normativer Aspekt ins Spiel, aber dessen Bezugspunkt ist nicht das Individuum, sondern der Staat, der mit seinen Gesetzen ein bestimmtes Ethos, bestimmte Denk- und Umgangsweisen unterstützt. An ihnen soll sich das Individuum orientieren oder hat sie eben zu akzeptieren, da sie in Gesetzen kodifiziert sind, so dass seine Selbstbestimmung hier begrenzt ist. Beispiele für eine solche Verschiebung von der individual- zur sozialethischen Betrachtungsweise mit Konsequenzen für die Begrenzung des Selbstbestimmungsrechts finden sich auch in Stellungnahmen des Ethikrats. So gab die Mehrheit des Ethikrats zu bedenken, eine Normalisierung der Suizidbeihilfe führe dazu, „den gesellschaftlichen Respekt vor dem Leben zu schwächen, fremdbestimmte Einflussnahmen in Situationen prekärer Selbstbestimmung zu begünstigen sowie Anstrengungen der Suizidprävention zu konterkarieren“ (Ethikrat 2017, 2). Die Beschränkung des Selbstbestimmungsrechts wird hier mit dem Verweis auf Werte gerechtfertigt, die in der Gesellschaft generell anerkannt sind oder sein sollten, wobei die oben bereits angesprochenen Folgenargumente angeführt werden. Der Ethikrat verweist in diesem Zusammenhang auf seine Stellungnahme von 2014 (Ethikrat 2014), die dem Bundestag als Grundlage für Erarbeitung des §217 diente. Indem es diesen Paragrafen für verfassungswidrig erklärte, hat das Bundesverfassungsgericht auch dem Votum der Mehrheit der Mitglieder des Ethikrats widersprochen.

\section{(3)}

Von einer Debatte, in der die eine Seite den Vorrang des Selbstbestimmungsrechts verteidigt, während die andere Seite nicht müde wird, vor den Folgen der Normalisierung einer höchstens in Ausnahmefällen akzeptablen Praxis zu warnen, sind keine weiterführenden Einsichten zu erwarten. Deshalb ist eine Beschreibung der strittigen Punkte erforderlich, die erkennen lässt, in welcher Richtung ein Ausweg aus dem argumentativen Patt zu suchen ist. Einen Ansatzpunkt dafür bietet die Analyse der amerikanischen Kontroverse um Abtreibung und Euthanasie, ${ }^{8}$ die Ronald Dworkin bereits vor einigen

8 Im Kontext der angelsächsischen Philosophie ist unter Euthanasie natürlich nicht das nationalsozialistische Programm der Ermordung kranker Mitmenschen zu verstehen. 
Jahren vorgelegt hat (Dworkin 1994). Seine Überlegungen hat er in seinem letzten großen Buch in einen weiteren Begründungskontext eingeordnet und wichtige Voraussetzungen geklärt (Dworkin 2014).

\section{(a) Der Grund moralischer Konflikte}

In der Auseinandersetzung zwischen den „liberalen“ Befürwortern der Zulässigkeit von Abtreibung bzw. Euthanasie und ihren „konservativen“ Gegnern vertritt Dworkin mit Nachdruck die liberale Position, aber er interessiert sich vor allem für die Struktur und für die Gründe des Konflikts, den er über weite Strecken mit der Distanz des neutralen Beobachters darstellt und analysiert. Entgegen dem ersten Anschein liegt die Wurzel des Konflikts, so Dworkins zentrale These, nicht in einer Kollision von Werten. Moralische Konflikte seien vielmehr als ,substantielle Folge einer tieferliegenden Einheit der Werte zu verstehen" (Dworkin 2014, 30), sie haben ihren Grund nicht in einem Wertekonflikt, sondern in einer unterschiedlichen Interpretation der Werte. Das Verhältnis von Begründungs- und Anwendungsebene moralischer Argumentationen muss dementsprechend neu bestimmt werden. Folgt man den gängigen moralphilosophischen Ansätzen, haben Konflikte ihren Grund vor allem in einem Dissens darüber, welches der vorausgesetzten Prinzipien im Fall ihrer Nichtvereinbarkeit stärker zu gewichten ist. Das einzelne moralische Urteil wird als Folgerung aus Grundprinzipien in Kombination mit einer Beschreibung des in Frage stehenden Problems angesehen, so dass die Ursache konträrer Urteile in Widersprüchen oder Inkohärenzen auf der Ebene der Prinzipien zu suchen ist. Hingegen zieht Dworkin zwischen die Ebene der Werte und die der Urteile eine weitere Ebene ein, nämlich die der Interpretation der Werte. Im Fall eines Urteilskonflikts ist auf diese Ebene zurückzugehen und nach Unterschieden der Interpretation zu suchen, wobei über die akzeptierten Werte durchaus Konsens bestehen kann. Aufgrund der Unterscheidung zwischen der Geltung und der Interpretation der Werte kann Dworkin seine These von der „Einheit der Werte“ (Dworkin 2014, 204) aufrechterhalten. Konflikte resultieren daraus, dass die Kontrahenten dieselben Werte in Anspruch nehmen, aber die Wertbegriffe unterschiedlich interpretieren. Daher sind Begründungen moralischer Urteile „eine Form der Interpretation“" (Dworkin 2014, 268). Wertbegriffe wie Verantwortlichkeit oder Freiheit sind ,interpretative Begriffe“ (Dworkin 2014, 274) und daher unscharf, was aber die alltägliche Verständigung in den meisten Fällen nicht behindert. Wenn aber in einem konkreten Fall kein übereinstimmendes Urteil erreicht wird, muss der Versuch unternommen werden, in „interpretati- 
ven Argumentationen“ (Dworkin 2014, 313) die Bedeutung der Wertbegriffe im Kontext anderer Wertbegriffe und akzeptierter Überzeugungen zu klären.

Dass diese Darstellung moralischer Konflikte und ihrer Gründe von starken und ungewöhnlichen Voraussetzungen abhängt, hebt Dworkin im Titel seines letzten großen Buches ausdrücklich hervor. Dort bezieht er sich auf einen Vers des griechischen Dichters Archilochos, dem zufolge die schlauen Füchse viele Sachen wissen, während der Igel nur eine, aber eine „große Sache“ weiß (Dworkin 2014, 13). Die „große Sache“ ist für Dworkin die Objektivität und die „metaphysische Unabhängigkeit“ (Dworkin 2014, 26, vgl. 207) der Werte. Sie sind „metaphysisch unabhängig“, weil sie nicht aus Annahmen über eine Realität jenseits der Welt der Wahrnehmungen und der sozialen Praktiken zu erklären sind. Entsprechend gibt es keine „nichtmoralische Erläuterung von moralischer Wahrheit“ und auch keine Metaethik, denn eine „Ontologie oder Epistemologie der Moral kann [...] nur innerhalb der Sphäre der Moral selbst ausgearbeitet werden“ (Dworkin 2014, 72). Werte können auch kein Gegenstand der Wahrnehmung oder der Intuition sein, weshalb Dworkin als Antwort auf die Frage, wie die Wahrheit eines moralischen oder ethischen Urteils festgestellt werden kann, nur auf die gängige Urteils- und Argumentationspraxis verweist.

\section{(b) Zwei Schritte zur Auflösung der Pattsituation}

Eine Auseinandersetzung mit den anspruchsvollen und weitreichenden Voraussetzungen von Dworkins Entwurf ist hier nicht möglich, aber im Hinblick auf das Argumentationsziel der vorliegenden Untersuchung auch nicht notwendig. Denn der Gedanke, den Grund moralischer Konflikte nicht auf der Ebene der Prinzipien zu suchen, sondern auf der Ebene der Interpretation, lässt sich als methodische Regel verstehen, deren Berechtigung sich aus den Resultaten ergibt, die auf diesem Weg gewonnen werden können. Von den Kontrahenten in einem moralischen Konflikt wird dieser Regel entsprechend zunächst verlangt, ihre Prämissen und Folgerungen explizit und nachvollziehbar darzulegen; auf diesem Weg können sie (mindestens) zu einem „rationalen Dissens“ (van den Daele 2008, 364) gelangen. Mit diesem Schritt sind die Kontrahenten zunächst einmal ihrer „moralischen Verantwortung“ (vgl. Dworkin 2014, 173) nachgekommen. Wenn es aber dabei bleibt, dass beide Seiten zur Verteidigung ihrer Position nur auf Prinzipien verweisen und betonen, dass sie hinter dieses „ethische Minimum“ nicht zurückgehen können, wird die Auseinandersetzung unproduktiv und eventuell abbrechen. Dies mag unausweichlich sein, wenn keinem der Kontrahenten noch 
überzeugende Argumente zur Verfügung stehen, so dass es beim „rationalen Dissens“ bleiben muss. Aber Dworkin zufolge sollen die Kontrahenten untersuchen, welche Werte es sind, auf die sie ihre Argumentation stützen und deren Geltung sie behaupten. Dabei kann sich herausstellen, dass sie sich durchaus auf dieselben Werte berufen, sie aber unterschiedlich interpretieren. Dies anzuerkennen setzt die Bereitschaft der Kontrahenten voraus, die eigene Interpretation als eine unter mehreren möglichen zu verstehen und eine Begründung dafür zu geben, weshalb die angesprochenen Werte so aufgefasst werden sollten, wie sie es jeweils vorschlagen.

Exemplarisch für das angegebene zweischrittige Verfahren ist Dworkins Analyse des Konflikts um die Zulässigkeit der Euthanasie, die er im siebenten Kapitel seines Buches „Die Grenzen des Lebens“ (Dworkin 1994, 247-301) vornimmt. In den ersten Kapiteln setzt sich Dworkin mit der Frage der Zulässigkeit der Abtreibung auseinander und überträgt die dabei eingeführten begrifflichen Unterscheidungen anschließend auf das Problem der Euthanasie. Den Ausgangspunkt bildet der Gedanke, dass sowohl Befürworter wie Gegner der Abtreibung bzw. der Euthanasie das menschliche Leben generell und „an sich“ für wertvoll halten (Dworkin 1994, 98f.). Auch über den Grundsatz, dass menschliches Leben nicht „vergeudet“ („frustriert“) (Dworkin 1994, 127) werden darf, lässt sich ein Konsens annehmen. Wenn dieser Grundsatz als kategorisches Verbot verstanden wird, gibt es keinen Spielraum für den Austausch von Argumenten über die Frage der Zulässigkeit der Abtreibung bzw. Euthanasie. Deren Gegner begründen ihre Position oft in der Sprache der Rechte und Interessen. Abtreibung sei grundsätzlich falsch, weil ein Embryo Rechte und Interessen hat, die zu respektieren sind. Diese „derivative“ Art der Begründung ist aber nur überzeugend, wenn die Prämisse des Besitzes von Rechten bzw. Interessen akzeptiert wird, woraus sich wiederum der unproduktive Streit um die Frage erklärt, $o b$ ein Embryo Interessen und Rechte hat. Um einen Ausweg aus dieser Pattsituation zu finden, schlägt Dworkin vor, bei der „originären“ Begründung anzusetzen, der zufolge menschliches Leben nicht vernichtet werden darf, weil es „heilig“ oder „unverletzlich“ ist. ${ }^{9}$ Es darf nicht zerstört oder „vergeudet“ werden, weil es das vorläufige Endstadium einer einmaligen, nicht reproduzierbaren Entwicklung bildet, sei diese Entwicklung primär natürlich-biologisch, wie im Fall der Entwicklung des Embryos, oder überwiegend biografisch, kulturell

9 Die Ausdrücke unverletzlich und heilig verwendet Dworkin als Synonyme (Dworkin 1994, 39; vgl. 270). 
und sozial, wie beispielsweise im Fall einer Person, die eine Ausbildung oder ein Studium erfolgreich absolviert und eine Konzeption für ihr zukünftiges Leben entwickelt hat. ${ }^{10}$ Die Rede von der Unverletzlichkeit des Lebens (im biologischen und biografischen Sinn) zeigt, dass das Leben nicht nur einen persönlichen Wert für die Person, die dieses Leben führt, sondern einen Wert an sich hat. Aus Dworkins Überlegungen ergibt sich also zunächst ein Argument gegen assistierten Suizid und Euthanasie (Dworkin 1994, 268f.). Aber wie mit der Unterscheidung zwischen biologisch und biografisch bereits angedeutet, kann der Begriff des Lebens in unterschiedlicher Weise akzentuiert werden. Wenn Leben nicht im juristisch-medizinischen, sondern im ethischen Sinn als „gutes“ bzw. "gelingendes“ Leben zum Thema wird, ${ }^{11}$ ändert sich die Perspektive der Beurteilung. Menschen messen das Gelingen ihres Lebens nicht nur an seiner Dauer oder an der Erfüllung ihrer Wünsche, sondern verfolgen auch wertebezogene Interessen (Dworkin 1994, 278f.); sie können kritisch beurteilen, worauf es ihnen im Leben ankommen sollte. Auf diese Weise kann ihr Leben an „innerer Stimmigkeit“ (Dworkin 1994, 284) gewinnen. Entsprechend droht der Verlust der Stimmigkeit des Lebens, wenn wichtige Aktivitäten nicht mehr möglich sind. Daher kann eine Person die Auffassung vertreten, die bereits erreichte Stimmigkeit ihres Lebens sei am besten dadurch zu bewahren, dass es zu einem Zeitpunkt beendet wird, der biografisch angemessen erscheint, auch wenn er im medizinischen Sinn vorzeitig ist. So kann es durchaus eine Schädigung sein, einen Menschen gegen dessen Willen am Leben zu halten. Daher sind Euthanasie und Suizidassistenz nicht grundsätzlich als Verletzung des Wertes des Lebens aufzufassen (Dworkin 1994, 300).

Dworkins Argumentation lässt sich als Begründung dafür verstehen, die gängige Auffassung von der Unverletzlichkeit des Lebens als eine von mehreren Interpretationen anzusehen. Die „konservativen“ Kritiker des

10 Dworkin spricht von einer „Investition in ein Menschenleben“, deren Komponenten „biologisch“ oder „menschlich“ sind (Dworkin 1994, 296). Die Ausdrücke Investition und auch Vergeudung führen dazu, die Argumentation aus utilitaristisch-ökonomischer Sicht zu verstehen, was irreführend wäre.

11 Dworkin unterscheidet zwischen dem „guten Leben“, das auch von zufälligen Widerfahrnissen abhängig ist, und der "gelungenen Lebensführung", die sich u. a. daran misst, ob die betreffende Person ihrer ethischen Verantwortung nachgekommen ist, das eigene Leben ernst zu nehmen und anzuerkennen, dass sein Gelingen (auch) unter objektiven Gesichtspunkten von Bedeutung ist (Dworkin 2014, 338-342). 
Verfassungsgerichtsurteils sollten sich durch seine Argumentation davon überzeugen lassen, dass ihre liberalen Gegner durchaus den Wert der Unverletzlichkeit des Lebens anerkennen. Die Meinungen gehen „nicht deshalb [auseinander], weil manche Menschen Werte geringschätzen, die andere hochhalten, sondern im Gegenteil deshalb, weil die in Frage stehenden Werte im Leben aller Menschen zentral sind“ (Dworkin 1994, 30of.).

Neu ist der Gedanke natürlich nicht, dass zur Analyse und Bewertung eines ethischen Urteils zweierlei gehört, nämlich einmal die Untersuchung, ob die zu beurteilende Handlung einem bestimmten Wert entspricht bzw. widerspricht, zum andern eine Antwort auf die sokratische „Was ist ...“-Frage, in welcher Bedeutung der gebrauchte Wertbegriff verwendet wird (vgl. Dworkin 2014, 313-316). Wenn aber die Gründe dafür zu suchen sind, weshalb der öffentliche ethische Diskurs über wichtige Fragen der Bioethik oder der medizinischen Ethik oft mit einem Dissens abbricht oder erst gar nicht in Gang kommt, bietet sich zur Erklärung in vielen Fällen die These an, der zweite der oben angegebenen Schritte sei gar nicht versucht worden. Ein Beispiel dafür liefert das Votum einiger Mitglieder des Ethikrats in einer Stellungnahme zur Präimplantationsdiagnostik, wenn sie abschließend feststellen, dass sich ihre Position „nicht auf eine von mehreren, pluralistisch nebeneinander stehenden ethischen Beurteilungsmöglichkeiten, sondern auf das ,ethische Minimum' stützt, das im Grundgesetz seinen Niederschlag gefunden hat“ (Ethikrat 2003, 101). Die Rede von einem „ethischen Minimum“ ist ambivalent. Entweder sind damit Überzeugungen gemeint, die eine Person nicht bereit ist aufzugeben. Oder es handelt sich um Grundprinzipien, die von allen Bürgerinnen und Bürgern anerkannt sein müssen, wenn ein Zusammenleben in einer gerechten Gesellschaft möglich sein soll. Der Verweis auf das Grundgesetz in dem angeführten Zitat deutet auf die zweite Verwendungsweise hin, aber der pragmatische Sinn der Äußerung besteht gerade darin klarzustellen, dass es den Unterzeichnern um die Unabänderlichkeit ihrer Überzeugungen geht und für sie eine Fortsetzung der Auseinandersetzung über diese Grenze hinweg nicht möglich ist. Dagegen ist einzuwenden, dass auch die zentralen Begriffe des Grundgesetzes mehrere Interpretationen zulassen, also durchaus eine weitere Auseinandersetzung möglich und sinnvoll ist. 


\section{(c) Autonomie und gelungenes Leben}

Mit dem jüngsten Urteil des Verfassungsgerichts wurde keine spezielle Lebensform befürwortet, auch wenn die Urteilskritiker dies mit ihren Warnungen vor einer Normalisierung des assistierten Suizids suggerieren. Vielmehr wurde das Spektrum der individuellen Handlungsoptionen ausgelotet und dessen Ausdehnung bestimmt, insbesondere durch den Grundsatz, dass eine Person weder der Gesellschaft noch dem Staat gegenüber rechenschaftspflichtig ist, wenn es um ihre eigenen Belange geht. Damit ist ein Freiraum eröffnet, der die Voraussetzung dafür bietet, die Frage nach dem gelungenen Leben erörtern zu können, ohne durch juridische oder gesellschaftliche Vorgaben in unzulässiger Weise eingeschränkt zu sein. Hier von „grenzenloser Autonomie“ zu reden, wäre missverständlich. Denn folgt man Dworkins Argumentation, ist es keine Sache der Willkür oder gar des Zufalls, welche Vorstellung vom gelungenen Leben eine Person übernommen hat. Vielmehr gehört es zu einer „gelungenen Lebensführung [...], sich um ein Leben zu bemühen, das nicht nur angenehm, sondern in einem kritischen Sinn gut ist“" (Dworkin 2014, 331). Dazu gehört unter anderem, die moralischen Verpflichtungen gegenüber anderen Personen zu respektieren, und daher verweisen Ethik und Moral wechselseitig aufeinander (Dworkin 2014, 342f.). Eine Verbindung zwischen beiden Disziplinen bildet der Begriff der Verantwortung, sei es (moralisch) gegenüber anderen oder (ethisch) in Bezug auf die eigene Person, sofern es um das Gelingen des eigenen Lebens geht. Dworkin plädiert dafür, dass der liberale Staat seinen Mitgliedern ihre „Eigenverantwortung“ (Dworkin 1994, 299) gerade dann zumutet, wenn es um zentrale Fragen ihres eigenen Lebens und ihres Lebensendes geht. Auch im Urteil des Verfassungsgerichts wird Selbstbestimmung in Kombination mit der Fähigkeit zur Verantwortung angeführt (BVerfG 2020, 61-63).

\section{(d) Verfassungsgericht und öffentlicher Vernunftgebrauch}

Das Verfassungsgericht soll, so führt Rawls aus, „der öffentlichen Vernunft gebührende anhaltende Wirksamkeit [...] verleihen“" (Rawls 1998, 338). Es dient als „exemplarische Instanz des öffentlichen Vernunftgebrauchs“ (Rawls 1998, 333), weil es grundlegende Fragen der Gerechtigkeit entscheidet und sich in seinen Begründungen nur auf die wesentlichen Verfassungsinhalte stützt, also von umfassenden religiösen oder philosophischen Lehren unabhängig ist. Natürlich beschreibt Rawls hier nicht die Realität, sondern ein Ideal. Aber dennoch macht es Sinn, die Entscheidung des Bundesverfas- 
sungsgerichts vom Februar 2020 zunächst einmal so aufzufassen, als wäre sie das Ergebnis eines öffentlichen Diskurses über einen der Grundsätze einer gerechten Gesellschaft. So hat ja das Verfassungsgericht einen Grundsatz hervorgehoben, nämlich das Prinzip des Vorrangs personaler Selbstbestimmung. Von Selbstbestimmung ist hier aber im rechtlich-politischen, nicht im moralisch-ethischen Sinn die Rede; es kann nicht Aufgabe eines Gerichts sein, die ethische Frage nach dem gelingenden Leben und der eigenen Verantwortung für dieses Gelingen zu beantworten. Soweit sie diese Unterscheidung übergeht, verfehlt eine Kritik an dem Urteil des Verfassungsgerichts von vornherein ihr Ziel.

\section{Literatur}

Braun, Matthias, Jens Ried und Peter Dabrock. 2015. „Die Kraft der Normalisierung. Ein evangelisch-theologischer Beitrag zur Regelung der ärztlichen Suizidassistenz", Zeitschrift für medizinische Ethik 61 (3): 216-229.

BVerfG (Bundesverfassungsgericht). 2020. Urteil des Zweiten Senats vom 26. Februar 2020 - 2 BvR 2347/15 -.

BVerwGE (Bundesverwaltungsgericht). 2017. Urteil vom 2.3.2017 - 3 C 19.15 -.

Dabrock, Peter. 2004a. „Fundamentaltheologische Bioethik angesichts der Herausforderungen moderner Gesellschaft“. In Menschenwürde und Lebensschutz, herausgegeben von P. Dabrock, L. Klinnert und S. Schardien, 19-56. Gütersloh: Gütersloher Verlagshaus.

Dabrock, Peter. 2004b. „Zum Verhältnis von Menschenwürde und Lebensschutz“. In Menschenwürde und Lebensschutz, herausgegeben von P. Dabrock, L. Klinnert und S. Schardien, 117-146. Gütersloh: Gütersloher Verlagshaus.

Dabrock, Peter. 2010. „,Leibliche Vernunft'. Zu einer Grundkategorie fundamentaltheologischer Bioethik und ihrer Auswirkung auf die Speziesismus-Debatte“. In Gattung Mensch. Interdisziplinäre Perspektiven, herausgegeben von P. Dabrock, R. Denkhaus und S. Schaede, 227-262. Tübingen: Mohr Siebeck.

Dabrock, Peter. 2015. „Selbstbestimmungsalternativen zwischen ethischer Bewertung und rechtlicher Normierung. Ein Beitrag (nicht nur) zur Sterbehilfe-Diskussion“. In Zeitschrift für Evangelische Ethik 59, 123-132.

Dabrock, Peter. 2020. „,Der Lebensschutz wiegt nichts.' Warum der Vorsitzende des Deutschen Ethikrats, der Theologe Peter Dabrock, den Karlsruher Spruch vehement ablehnt (Interview mit Matthias Drobinski)“. Süddeutsche Zeitung Nr. 49 vom 28.2.2020: 2.

Daele, Wolfgang van den. 2008. „Streitkultur. Über den Umgang mit unlösbaren moralischen Konflikten im Nationalen Ethikrat". In Politische Kultur im Wandel von Staatlichkeit, herausgegeben von D. Gosewinkel und G.F. Schuppert, 357-384. Berlin: edition sigma. 
Dreier, Horst. 2007. „Grenzen des Tötungsverbotes. Teil 2“. Juristenzeitung 62 (7): 317-326.

Dworkin, Ronald. 1994 [1993]. Die Grenzen des Lebens. Abtreibung, Euthanasie und persönliche Freiheit. Reinbek: Rowohlt.

Dworkin, Ronald. 2014 [2011]. Gerechtigkeit für Igel. Berlin: Suhrkamp.

Ethikrat, Nationaler. 2003. Genetische Diagnostik vor und während der Schwangerschaft, Berlin.

Ethikrat, Nationaler. 2006. Selbstbestimmung und Fürsorge am Lebensende. Berlin.

Ethikrat, Deutscher. 2014. Zur Regelung der Suizidbeihilfe in einer offenen Gesellschaft: Deutscher Ethikrat empfiehlt gesetzliche Stärkung der Suizidprävention. Berlin.

Ethikrat, Deutscher. 2017. Suizidprävention statt Suizidunterstützung. Erinnerung an eine Forderung des Deutschen Ethikrates anlässlich einer Entscheidung des Bundesverwaltungsgerichts. Berlin.

Gethmann, Carl Friedrich. 2020. „Ethische Beratung durch Ethikräte. Zeitschrift für Didaktik der Philosophie und Ethik 42 (1): 27-34.

Gutmann, Thomas, und Michael Quante. 2017. Menschenwürde, Selbstbestimmung und Pluralismus: Zwischen sittlicher Vorgabe und deontologischer Konstruktion, Archiv für Rechts- und Sozialphilosophie 103 (3): 322-336. https://doi. org/10.25162/arsp-2017-0239.

Quante, Michael. 2014. Menschenwürde und personale Autonomie. Demokratische Werte im Kontext der Lebenswissenschaften. 2. durchges. Aufl. Hamburg: Meiner.

Rawls, John. 1998 [1993]. Politischer Liberalismus. Frankfurt a.M.: Suhrkamp.

Rawls, John. 2003 [2001]. Gerechtigkeit als Fairneß. Ein Neuentwurf. Frankfurt a.M.: Suhrkamp.

Schardien, Stefanie. 2004. „Menschenwürde und Sterbehilfe - wider einfache Vorgaben“. In Menschenwürde und Lebensschutz, herausgegeben von P. Dabrock, L. Klinnert und S. Schardien, 289-316. Gütersloh: Gütersloher Verlagshaus. 